\title{
QUANTIZATION OF THE SPHERICALLY SYMMETRIC CONFIGURATION OF THE GRAVITATIONAL AND ELECTROMAGNETIC FIELDS
}

\author{
M.G. Holovko, V.D. Gladush \\ Dnipro National University, Gagarin Ave, 725014, Ukraine, \\ dilmendar@gmail.com,vgladush@gmail.com
}

\begin{abstract}
To construct a quantum model of a charged black hole $(\mathrm{CBH})$, we introduce a modified description of the configuration of the electromagnetic and gravitational fields in a spherically symmetric space-time, which consists of $\mathrm{T}$ - and $\mathrm{R}$ - regions. We choose such coordinate system that the desired metric functions depend only on the time coordinate in the T-region, and on the space coordinate in the R-region. Then, the initial action for the configuration decays into terms which describe the fields in the T- and R-regions with the time and the space evolutionary coordinate respectively. We define new coordinates in the R- and T- regions, what allows us to unify the form of the Lagrangians, in each of them and carry out their uniform analysis. Then we construct the canonical formalism for obtained degenerate system according to the method of D.M.Gitman and I.V.Tyutin. It appears that system contains non-physical degrees of freedom. For their expicit separation we carry out the canonical transformation to new canonical variables. In these variables the constraints are reduced to the canonical form and physical part of the Hamilton function of the system is identically equal to zero. This leads to the fact that the desired wave function is determined only by the eigenvalue equations for the operators of observable physical quantities. According that considered system has only two observables - charge and mass of black hole - for further construction of quantum model of this system we introduce its mass and charge functions and find their expression in the new canonical variables. The solution of eigenvalue equations for corresponding operators leads to continuous spectra of charge and mass in considered model of CBH.
\end{abstract}

Keywords: black holes, mass function, charge function, Hamiltonian constraint, quantization, mass and charge operators.

\section{Introduction}

Spherically symmetric configurations of gravitational and other fields are the simplest models for testing the basic ideas and consequences of quantum gravity. They represent a convenient ground for studying some of the problems that arise at strict consideration of more complete theory. One of the main features of general-relativistic configurations is their degeneracy. The general formalism of the canonical approach to degenerate systems was constructed by Dirac (1979), which was developed in further works (see, for example, Sundermeyer, 1982; Nesterenko, 1986; Gitman, 1986; etc.). The problems of the classical and quantum description of spherically symmetric configurations of the gravitational and electromagnetic fields in the framework of the canonical approach were considered in many papers. A general, geometrodynamic approach to a spherically symmetric gravitational field was developed by Kuchar K. (1996), which was then generalized to the case of a spherically symmetric electromagnetic and gravitational configuration (Louko, 1996; Vaz, 2000). The proposed model is based on the observation that classical spherically symmetric configurations of electromagnetic and gravitational fields are stationary from the point of view of an external observer and have certain regions of space-time with dynamic behavior (T-regions). This means that these regions do not allow the time-like Killing vector, which implies in them the evolution of the space-time geometry in time. This evolution of space-time geometry is responsible for the quantum-mechanical properties of the model of a BH. Such models, with a fixed evolutionary time coordinate and a spacelike Killing vector, were considered in several works (Nakamura, 1993; Gladush, 2016; Holovko, 2017). In this paper constructed a quantum model of a charged black hole according to the method of D.M.Gitman and I.V.Tyutin (1986). 


\section{Classic description of $\mathrm{CBH}$}

The action for the gravitational and electromagnetic fields in space-time $V^{(4)}$ has the form

$$
S_{t o t}=-\frac{1}{16 \pi} \int_{V^{(4)}}\left(\frac{c^{3}}{\kappa} R^{(4)}+\frac{1}{c} F_{\mu \nu} F^{\mu \nu}\right) \sqrt{-g^{(4)}} d^{4} x .
$$

For a spherically symmetric configuration, the electromagnetic field tensor and the interval have the forms

$$
F_{\mu \nu}=A_{\nu, \mu}-A_{\mu, \nu} \rightarrow F_{a b}=A_{b, a}-A_{a, b},
$$

$d s^{2}=h\left(x^{0}, r\right)\left(d x^{0}\right)^{2}+g\left(x^{0}, r\right) d r^{2}+R^{2}\left(x^{0}, r\right) d \sigma^{2}$,

where $d \sigma^{2}=d \theta^{2}+\sin \theta d \alpha^{2}$ is the angular part of the metric; $\mathrm{a}, \mathrm{b}=0,1$. After integrating over the angles and discarding the surface term, the action (1) can be reduced to the form

$$
\begin{aligned}
S & =\int_{V^{2}}\left(\frac { c ^ { 3 } } { 2 \kappa } \sqrt { g h } \left[1+\frac{R R_{, 1}}{g}(\ln R h)_{, 1}-\right.\right. \\
& \left.\left.-\frac{R R_{, 0}}{h}(\ln (g R))_{, 0}\right]+\frac{1}{2 c} \frac{\left(A_{1,0}-A_{0,1}\right)^{2} R^{2}}{\sqrt{g h}}\right) d x^{2}
\end{aligned}
$$

Here $X_{, 0} \equiv \partial X / \partial x^{0}, X_{, 1} \equiv \partial X / \partial r$ denote the derivatives with respect to $x^{0}$ and $x^{1}$. Information on the structure of space is contained in the quantity $(\nabla R)^{2}=$ $g^{a b} R_{, a} R_{, b}$ The surface $R\left(x^{0}, r\right)=R_{g}=$ const for which $(\nabla R)^{2}=0$ divides $V^{4}$ into one T- and two Rregions. Moreover, $(\nabla R)^{2}>0$ in the T-region, and $(\nabla R)^{2}<0$ in the R-region. Using the generalized Birkhoff theorem, we can choose a coordinate system in the R-region in which $\mathrm{h}, \mathrm{g}$ and $\mathrm{R}$ depend only on the spacelike coordinate $\mathrm{r}$. Similarly, in the T-region there exists a coordinate system in which $\mathrm{h}, \mathrm{g}$ and $\mathrm{R}$ depend only on the timelike coordinate $x^{0}$. Then the metrics in the $\mathrm{R}$ - and T-regions take the form:

$$
\begin{gathered}
d s_{R}^{2}=h(r)\left(d x^{0}\right)^{2}+g(r) d r^{2}+R^{2}(r) d \sigma^{2}, \\
d s_{T}^{2}=h\left(x^{0}\right)\left(d x^{0}\right)^{2}+g\left(x^{0}\right) d r^{2}+R^{2}\left(x^{0}\right) d \sigma^{2} .
\end{gathered}
$$

In this case, the action (4) is divided in the sum $S=S_{R}+S_{T}$, where $S_{R}$ and $S_{T}$ are the actions defined in the $\mathrm{R}$ and $\mathrm{T}$ regions respectively. The Lagrangians corresponding to them have the form

$$
\begin{aligned}
& L_{R}=\frac{\lambda_{T}}{2 c} \frac{A_{0,1}^{2} R^{2}}{\sqrt{g h}}+\frac{\lambda_{T} c^{3}}{\kappa} \sqrt{g h}\left[1+\frac{R R_{, 1}}{g}(\ln R h)_{, 1}\right], \\
& L_{T}=\frac{\lambda_{R}}{2 c} \frac{A_{1,0}^{2} R^{2}}{\sqrt{g h}}+\frac{\lambda_{R} c^{3}}{\kappa} \sqrt{g h}\left[1-\frac{R R_{, 0}}{h}(\ln R g)_{, 0}\right] .
\end{aligned}
$$

Here $\lambda_{T}$ and $\lambda_{R}$ - constants, obtained by integration of te action over coordinate $x^{0}$ in R-region and coordinate $\mathrm{r}$ in T-region. Since the Lagrangian is defined up to a constant factor, we further assume that $\lambda_{R}=\lambda_{T}=1$ (meter).

It is convenient to introduce new variables in the $\mathrm{R}$ and T- regions:

$$
\begin{array}{rlrl}
F_{R} & =-A_{0}, & U_{R}=R h, & N_{R}=\sqrt{g h}, \\
F_{T}=A_{1}, & U_{T}=-R g, & N_{T}=\sqrt{g h} .
\end{array}
$$

In these variables, the Lagrangians (7) and (8) take a uniform form:

$$
L=\frac{F_{, \alpha}^{2} R^{2}}{2 c N}+s N\left(1+\frac{R_{, \alpha} U_{\alpha}}{N^{2}}\right) .
$$

where $s=c^{3} / \kappa, \alpha-$ is the evolutionary parameter, which in each of the regions takes the form $\alpha=x^{0}=c t$ or $\alpha=r$ respectively. For the considered configuration (10), the Hessian is zero. Therefore, the system is degenerate and the construction of the canonical theory is specific. In order to construct the canonical formalism for the considered dynamical system, we use the method of D.M.Gitman and I.V.Tyutin. We introduce an extended system of Lagrange equations for the system:

$$
\dot{Q}^{i}=V^{i}, \quad M_{i j} \dot{V}^{j}=K_{i}, \quad P_{i}=\frac{\partial L}{\partial V^{i}},
$$

Here $Q^{i}, V^{i}, P_{i}$ are the sets of generalized coordinates $(F, R, U, N)$, velocities $\left(V_{F}, V_{R}, V_{U}, V_{N}\right)$ and momenta $\left(P_{F}, P_{R}, P_{U}, P_{N}\right)$

$$
M_{i j}=\frac{\partial^{2} L}{\partial V^{i} \partial V^{j}},
$$

- the Hessian matrix of the system, and the quantities $K_{i}$ are defined as follows:

$$
K_{i}=\frac{\partial L}{\partial Q^{i}}-\frac{\partial^{2} L}{\partial V^{i} \partial Q^{j}} V^{j}
$$

The Hamiltonian function for the extended Hamiltonian system is introduced as follows:

$$
H=P_{i} V^{i}-L^{V}
$$

From equations $P_{i}=\partial L / \partial V^{i}$ we obtain expressions for the velocities $V_{F}, V_{R}, V_{U}$, and also the primary constraint $P_{N}=0$. Demanding its preservation during the evolution of the system, we get a secondary constraint:

$$
0=\left\{P_{N}, H\right\}=-\frac{1}{s}\left(P_{U} P_{R}-s^{2}\right)-\frac{c}{2 R^{2}} P_{F}^{2} .
$$

The obtained constraint remains identically, and therefore no new constraints arise. The Poisson brackets of the found constraints are also equal to zero, so both of them are first-type constraints. This means that the Hamilton function contains non-physical degrees of freedom. For their explicit separation, we can make a canonical transformation to new canonical variables 
$\left(q^{i}, p_{i}\right)$ with the following generating function of the second type:

$$
\begin{gathered}
G_{2}\left(\alpha, Q^{i}, p_{i}\right)=F p_{1}+\left(R-\frac{p_{2}}{s}\right) p_{3}+ \\
+2 \sqrt{s^{2} R U+\frac{c s U}{2 R}-s U p_{2}}+N p_{4} .
\end{gathered}
$$

Herefrom for the new canonical variables we obtain:

$$
\begin{gathered}
q^{1}=F+\frac{s c}{R} \frac{P_{F}}{P_{U}}, \quad q^{2}=-\left(\frac{P_{R}}{s}+\frac{c}{R^{2}} \frac{P_{F}^{2}}{P_{U}}\right), \\
q^{3}=\frac{U P_{U}^{2}}{s^{2}}-\frac{c P_{F}^{2}}{2 s R}, \quad q^{4}=N ; \\
p_{1}=P_{F}, \quad p_{2}=s\left(R-\frac{U P_{U}^{2}}{s^{2}}\right), \\
p_{3}=P_{R}-\frac{s^{2}}{P_{U}}+\frac{c s}{R^{2}} \frac{P_{F}^{2}}{P_{U}}, \quad p_{4}=P_{N} .
\end{gathered}
$$

Old canonical variables are expressed in terms of new variables as follows:

$$
\begin{gathered}
F=q^{1}+p_{1} \frac{p_{3}+s q^{2}}{p_{2}+s q^{3}}, \quad R=q^{3}+\frac{p_{2}}{s} \\
U=\left(\frac{p_{3}+s q^{2}}{s}\right)^{2}\left(q^{3}+\frac{c p_{1}^{2}}{2\left(p_{2}+s q^{3}\right)}\right), \quad N=q^{4} \\
P_{F}=p_{1}, \quad P_{R}=s\left(\frac{c p_{1}^{2}\left(p_{3}+s q^{2}\right)}{2\left(p_{2}+s q^{3}\right)^{2}}-q^{2}\right), \\
P_{U}=-\frac{s^{2}}{p_{3}+s q^{2}}, \quad P_{N}=p_{4} .
\end{gathered}
$$

In the new variables, the Hamilton function and the constraints take the form:

$$
\begin{gathered}
H=p_{4} V_{N}-\frac{s q^{4} p_{3}}{p_{3}+s q^{2}}, \\
p_{3}=0, \quad p_{4}=0 .
\end{gathered}
$$

From the fact that in new variables the constraints have the canonical form, it means that the physical degrees of freedom are contained in the pairs of quantities $\left(q^{1}, p_{1}\right)$ and $\left(q^{2}, p_{2}\right)$.

\section{Quantum description of $\mathrm{CBH}$}

Assuming for the Hamiltonian (19) that the constraints (20) are equal to zero, we find that the physical part of the Hamiltonian function is identically equal to zero. Therefore, for a quantum description of the system, its wave function is determined only by the eigenvalue equations for the operators of the observed physical quantities. For the considered system there are only two quantities: the mass and charge of the black hole. In the classical case, the charge is determined by the charge function as follows:

$$
Z=\frac{R^{2}}{\sqrt{g h}}\left(A_{0,1}-A_{1,0}\right)
$$

The total mass of the configuration has the form

$$
M=\frac{s}{c} R\left(1+\frac{R_{, 0}^{2}}{h}-\frac{R_{, 1}^{2}}{g}\right)+\frac{Z^{2}}{2 c^{2} R} .
$$

It has the meaning of the total field mass of a spherical region of radius $\mathrm{R}$ of the configuration in question, taking into account the contribution of the electromagnetic field. When changing to new coordinates in the $\mathrm{R}$ - and T-regions, and then transit to new canonical variables, we find:

$$
M=\frac{p_{1}}{c}, \quad Z=c p_{2}
$$

Then the corresponding operators of mass and charge will have the form:

$$
\hat{M}=-\frac{i \hbar}{c} \frac{\partial}{\partial q^{1}}, \quad \hat{Z}=-i \hbar c \frac{\partial}{\partial q^{2}} .
$$

For the wave function of the configuration, it is necessary to solve the system of equations:

$$
\left\{\begin{aligned}
i \hbar \frac{\partial \Psi}{\partial \alpha} & =\hat{H} \Psi \\
\hat{M} \Psi & =m \Psi \\
\hat{Z} \Psi & =z \Psi
\end{aligned}\right.
$$

Taking into account that the physical degrees of freedom are only described by pairs of canonical variables $\left(q^{1}, p_{1}\right)$ and $\left(q^{2}, p_{2}\right)$, we get:

$$
\Psi=\Psi\left(q^{1}, q^{2}\right)=C \exp \frac{i}{\hbar}\left[m c q^{2}+\frac{s q^{1}}{c}\right] .
$$

Since this system has a solution for any values of $m$ and $\mathrm{z}$, the mass and the charge spectra of $\mathrm{BH}$ are continuous.

\section{Conclusion}

In the previous work of one of the authors (Gladush V., see the article in this collection), there was carried out a direct quantization of the constraints for the considered system. In this paper, according to the method of Gitman and Tyutin, quantization of considered system was carried out after separation of physical and non-physical degrees of freedom by suitable canonical transformation. This approach is consistent with the results of the geometrodynamic approach (Louko, 1996; Vaz., 2000). In the case of non-charged $\mathrm{BH}(\mathrm{z}=0)$, the result obtained is analogous to the result for the Schwarzschild black hole of the general geometrodynamic approach (Kuchar, 1996).

\section{References}

Dirac P.: 1979, Lectures on quantum mechanics, in Principles of quantum mechanics, (Nauka, Moscow). Sundermeyer K.: 1982, Lec. Not. Phys., V.169.

Nesterenko V.V, Chervyakov A.M.: 1986, Singular Lagrangians, in Classical dynamics and quantization, (JINR, Dubna), 102. 
Gitman D., Tyutin I.: 1986, Canonical quantization Gladush V.: 2016, Visn. Dnipr. univ., $24,31$. of fields with constraints, (Nauka, Moscow), 216.

Kuchar K.: 1994, Phys. Rev., D50, 3961.

Louko J., Winters-Hilt S.: 1996, Phys. Rev. D, 54, 2647.

Holovko M., Gladush. V.: 2017, Quantization of spherically symmetric gravitational field. International Conference "Astronomy and Space Physics", Kiev, 2017, 31.

Vaz C., Witten L.: 2000, Phys. Rev. D, 63, 24.

Nakamura K., Konno S., Oshiro Y.: 1993, Prog. Theor. Phys., 90, 861. 